\title{
Novel Ag@Nitrogen-doped Porous Carbon Composite with High Electrochemical Performance as Anode Materials for Lithium-ion Batteries
}

\author{
Yuqing Chen ${ }^{1} \cdot$ Jintang $\mathrm{Li}^{1} \cdot$ Guanghui Yue ${ }^{2} \cdot$ Xuetao Luo $^{1}$
}

Received: 5 December 2016/ Accepted: 15 January 2017/Published online: 18 February 2017

(C) The Author(s) 2017. This article is published with open access at Springerlink.com

\section{Highlights}

- A novel Ag@nitrogen-doped porous carbon (Ag-NPC) composite was applied to lithium-ion batteries. The encapsulation of Ag nanoparticles (Ag NPs) into NPC boosts reversible capacity from 501.6 to $852 \mathrm{mAh} \mathrm{g}^{-1}$.

- Ag-NPC shows a much better cycling performance than NPC due to the synergistic effect of NPC and Ag NPs.

\begin{abstract}
A novel Ag@nitrogen-doped porous carbon (Ag-NPC) composite was synthesized via a facile hydrothermal method and applied as an anode material in lithium-ion batteries (LIBs). Using this method, $\mathrm{Ag}$ nanoparticles (Ag NPs) were embedded in NPC through thermal decomposition of $\mathrm{AgNO}_{3}$ in the pores of NPC. The reversible capacity of Ag-NPC remained at $852 \mathrm{mAh} \mathrm{g}^{-1}$ after 200 cycles at a current density of $0.1 \mathrm{~A} \mathrm{~g}^{-1}$, showing its remarkable cycling stability. The enhancement of the electrochemical properties such as cycling performance, reversible capacity and rate performance of Ag-NPC compared to the NPC contributed to the synergistic effects between Ag NPs and NPC.
\end{abstract}

Xuetao Luo

xuetao@xmu.edu.cn

1 Fujian Key Laboratory of Advanced Materials, Department of Materials Science and Engineering, College of Materials, Xiamen University, Xiamen 361005, People's Republic of China

2 Department of Materials Science and Engineering, College of Materials, Xiamen University, Xiamen, 361005, People's Republic of China

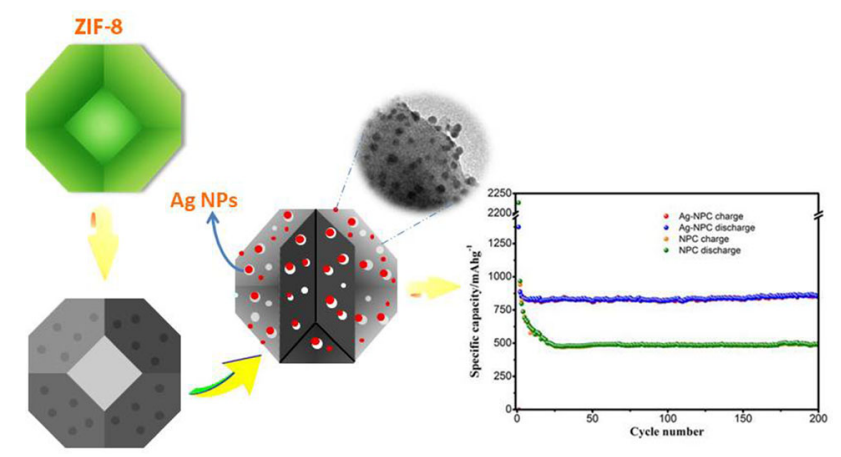

Keywords Nitrogen-doped porous carbon $\cdot \mathrm{Ag}$ nanoparticles $\cdot$ Synergistic effects $\cdot$ Lithium-ion batteries

\section{Introduction}

In recent years, lithium-ion batteries (LIBs) have not only been widely used for consumer electronics, but have also proved promising for electric vehicles, owing to their unique advantages, such as high energy and power density, no memory effect and environmental friendliness [1-3]. Currently, graphitic materials are the most commonly used commercial anode materials for LIBs by virtue of their 
superior cycling stability and high coulombic efficiency [4]. However, due to quite a low theoretical capacity of $372 \mathrm{mAh} \mathrm{g}^{-1}$, it would be hard for graphite to meet increasingly high energy requirements in electric vehicles [5]. A variety of materials have been exploited as anode materials for LIBs in the past decades, such as transitionmetal oxides [6-8], and silicon-based [9-11] or tin-based [12-14] materials, which have ultra-high theoretical capacity. Unfortunately, these materials react with lithium and are more inclined to form $\mathrm{Li}_{2} \mathrm{O}$ than $\mathrm{Li}-\mathrm{M}$ alloys. Due to the fact that it is an electrochemically irreversible reaction, it results in a large irreversible capacity [15]. Silver is an appealing option for anode materials, owing to its relatively high specific capacity, which is attributed to the formation of several $\mathrm{Ag}$-Li alloys (up to $\mathrm{AgLi}{ }_{12}$ ) within a very low voltage range $(0.25-0 \mathrm{~V})$ [16]. Moreover, silver has the best electrical conductivity among all metals and excellent lithium diffusivity, which can efficiently boost the electrochemical performance [17]. However, silver also suffers from undesirable volumetric expansion during lithium insertion. To alleviate this harmful effect, different strategies have been developed, such as downsizing the particle dimensions to the nanoscale, introducing a porous structure to the solid particles and designing silver-containing composites [18-20]. Carbon is a common matrix for silver. Shilpa et al. used hollow carbon nanofibers as a buffer matrix and embedded silver nanoparticles in them through the coaxial electrospinning method [15]. Hsieh et al. dispersed silver nanorods onto graphene nanosheets by the hydrothermal method [21].

Metal organic frameworks (MOFs) have been attracting increasing attention as carbon sources for anode materials because various types of MOF precursors can result in derived carbon with a uniform, controllable, porous structure and enable innate doping of heteroatoms [22-24]. On the basis of previous research, the nanopores can facilitate rapid electrolyte transfer [25]. In addition, the heteroatomdoped carbon always performs at a higher specific capacity and outstanding cycling stability compared to the nondoped carbon [26-29]. Song et al. prepared a cage-like carbon/nano-Si composite as anode materials by the template method to embed Si nanoparticles into ZIF-8. The resulting nano-Si/C composite showed a higher reversible capacity than many $\mathrm{Si} / \mathrm{C}$ composites previously reported [30]. Xie et al. fabricated a sandwich-like, graphene-based, porous nitrogen-doped carbon (PNCs@Gr) through the pyrolysis of zeolitic imidazolate framework nanoparticles grown in situ on GO (ZIF-8@GO), which exhibited outstanding electrochemical performance among carbonaceous materials used as anode materials [31].

We used ZIF-8-derived carbon as a matrix for silver nanoparticles (Ag NPs), which can provide not only rigid matrices with nanopores, but also a relatively high nitrogen content. We designed a strategy to incorporate Ag NPs into $\mathrm{N}$-doped porous carbon uniformly via a facile hydrothermal method without any reduction agent. When applied as the anode material for the Li-ion battery, the Ag-NPC showed excellent electrochemical performance over bare NPC, which was attributed to the synergistic effect of Ag NPs and the carbon matrix.

\section{Experimental}

\subsection{Chemicals}

Methanol $\left(\mathrm{CH}_{3} \mathrm{OH}\right.$, Sinopharm Chemical Reagent Co. Ltd, $>99.5 \%)$ 2-methylimidazole $\left(\mathrm{C}_{4} \mathrm{H}_{6} \mathrm{~N}_{2}, \quad\right.$ Sinopharm Chemical Reagent Co. Ltd., 99\%), zinc nitrate $\left(\mathrm{Zn}\left(\mathrm{NO}_{3}\right)_{2} \cdot 6 \mathrm{H}_{2} \mathrm{O}\right.$, Shanghai Titanchem Co. Ltd., $\left.>99.8 \%\right)$, 1-methylimidazole $\left(\mathrm{C}_{4} \mathrm{H}_{6} \mathrm{~N}_{2}\right.$, Sinopharm Chemical Reagent Co. Ltd., 99\%) and silver nitrate $\left(\mathrm{AgNO}_{3}\right.$, Sinopharm Chemical Reagent Co. Ltd., $>99.8 \%$ ) were used. All reagents were used without further purification.

\subsection{Preparation of N-doped Porous Carbon (NPC)}

ZIF-8 was synthesized according to method reported in the literature [32]. Specifically, a methanolic solution $(400 \mathrm{~mL})$ of 2-methylimidazole $(6.48 \mathrm{~g})$ and 1-methylimidazole $(6.28 \mathrm{~mL})$ was quickly poured into a methanolic solution $(400 \mathrm{~mL})$ of $\mathrm{Zn}\left(\mathrm{NO}_{3}\right)_{2} \cdot 6 \mathrm{H}_{2} \mathrm{O}(5.88 \mathrm{~g})$ and stirred for $2 \mathrm{~min}$ and then kept still for $16 \mathrm{~h}$. After that, the solution was centrifuged, washed by methanol and dried at $60{ }^{\circ} \mathrm{C}$ for $3 \mathrm{~h}$ to produce a white solid (ZIF-8). Then, the solid was ground into powder, followed by heat treatment at $800{ }^{\circ} \mathrm{C}$ for $5 \mathrm{~h}$ under an argon atmosphere. After letting it cooldown to the room temperature, the obtained product was dispersed into an $\mathrm{HCl}$ solution $(100 \mathrm{~mL}, 20 \mathrm{wt} \%$ in water) and stirred for $24 \mathrm{~h}$ to remove residual metallic $\mathrm{Zn}$ and/or $\mathrm{ZnO}$. The mixture was then washed thoroughly with distilled water several times until all the zinc ions were removed. Finally, the resultant product was dried at $60{ }^{\circ} \mathrm{C}$ in vacuum oven for $6 \mathrm{~h}$ to obtain an $\mathrm{N}$-doped hierarchically porous carbon.

\subsection{Synthesis of Ag-NPC Composite}

The Ag-NPC composite was synthesized by a facile hydrothermal process without a reduction agent [33]. The NPC (100 mg) was quickly dispersed into an aqueous solution of $\mathrm{AgNO}_{3}\left(25 \mathrm{~mL}, 20 \mathrm{mmol} \mathrm{L}{ }^{-1}\right)$, and the mixture was subsequently homogenized by intensely stirring it for $1 \mathrm{~h}$ in the dark. Then, the aqueous suspension was heated at $100{ }^{\circ} \mathrm{C}$ while gently stirring it under argon flow for $10 \mathrm{~min}$, followed by quickly cooling it with running 
water. Next, the mixture was centrifuged and washed with distilled water several times and then dried at $60{ }^{\circ} \mathrm{C}$ in a vacuum oven for $5 \mathrm{~h}$.

\subsection{Characterization}

Powder X-ray diffraction (XRD) analysis was performed using a Bruker-Axs D8 Advance X-ray diffractometer with $\mathrm{Cu}$ Ka radiation $(\lambda=0.15406 \mathrm{~nm})$. The morphology of the sample was studied using a Hitachi SU70 field emission scanning electron microscope (SEM) at $10 \mathrm{kV}$. The highresolution transmission electron microscopy (HRTEM) characterization was performed on a Tecnai F30 microscope at an accelerating voltage of $300 \mathrm{kV}$. The specific surface area and pore size distribution were analyzed by using a TriStar II 3020. The thermogravimetric analysis (TGA) was carried out on a SDTQ600 thermoanalyzer in air. The elemental analysis was performed using a Vario ELIII. The X-ray photoelectron spectroscopy (XPS) was performed using a Thermo Scientific ESCALAB 250Xi with $\mathrm{Al} K \alpha$ radiation $(h v=1486.8 \mathrm{eV})$.

\subsection{Electrochemical Measurements}

The active materials, NPC or Ag-NPC composite (70 $\mathrm{wt} \%$ ), acetylene black (20 wt $\%$ ) and poly(vinyl difluoride) (PVDF, $10 \mathrm{wt} \%$ ) were diffused in 1-methyl-2pyrrolidinone (NMP) and stirred intensely to form a homogeneous slurry. Then, a copper foil was coated with the slurry and dried in a vacuum oven at $80{ }^{\circ} \mathrm{C}$ for $12 \mathrm{~h}$. The lithium foil was used as the counter electrode as well as the reference electrode. A polypropylene membrane and $\mathrm{LiPF}_{6}$ were used as the separator and electrolyte, respectively. The galvanostatic discharge/charge experiments were performed on a Neware battery tester. Cyclic voltammetry $(\mathrm{CV})$ and electrochemical impedance spectroscopy (EIS) measurements were carried out on an Autolab electrochemical workstation (NOVA 1.9).

\section{Results and Discussion}

The morphology of the ZIF-8 and derived NPC was detected using a SEM. The ZIF-8 precursor showed an average particle size of about $2 \mu \mathrm{m}$ (Fig. 1a) and its typical rhombic dodecahedron morphology (Fig. 1b). Notably, after direct carbonization at $800{ }^{\circ} \mathrm{C}$ for $5 \mathrm{~h}$ in argon flow, the NPC well inherited the morphology of the ZIF-8 precursor with no evident structure collapse (Fig. 1c). And the surface of NPC appears smoother than that of ZIF-8 precursor (Fig. 1d). The elemental mapping provided evidence of the presence of $\mathrm{N}$ (Fig. 1e), and the nitrogen content was about $17 \mathrm{wt} \%$, according to the elemental analysis. The elemental mapping of Zn (Fig. 1f) showed that zinc or zinc oxide species were completely removed after acid pickling.
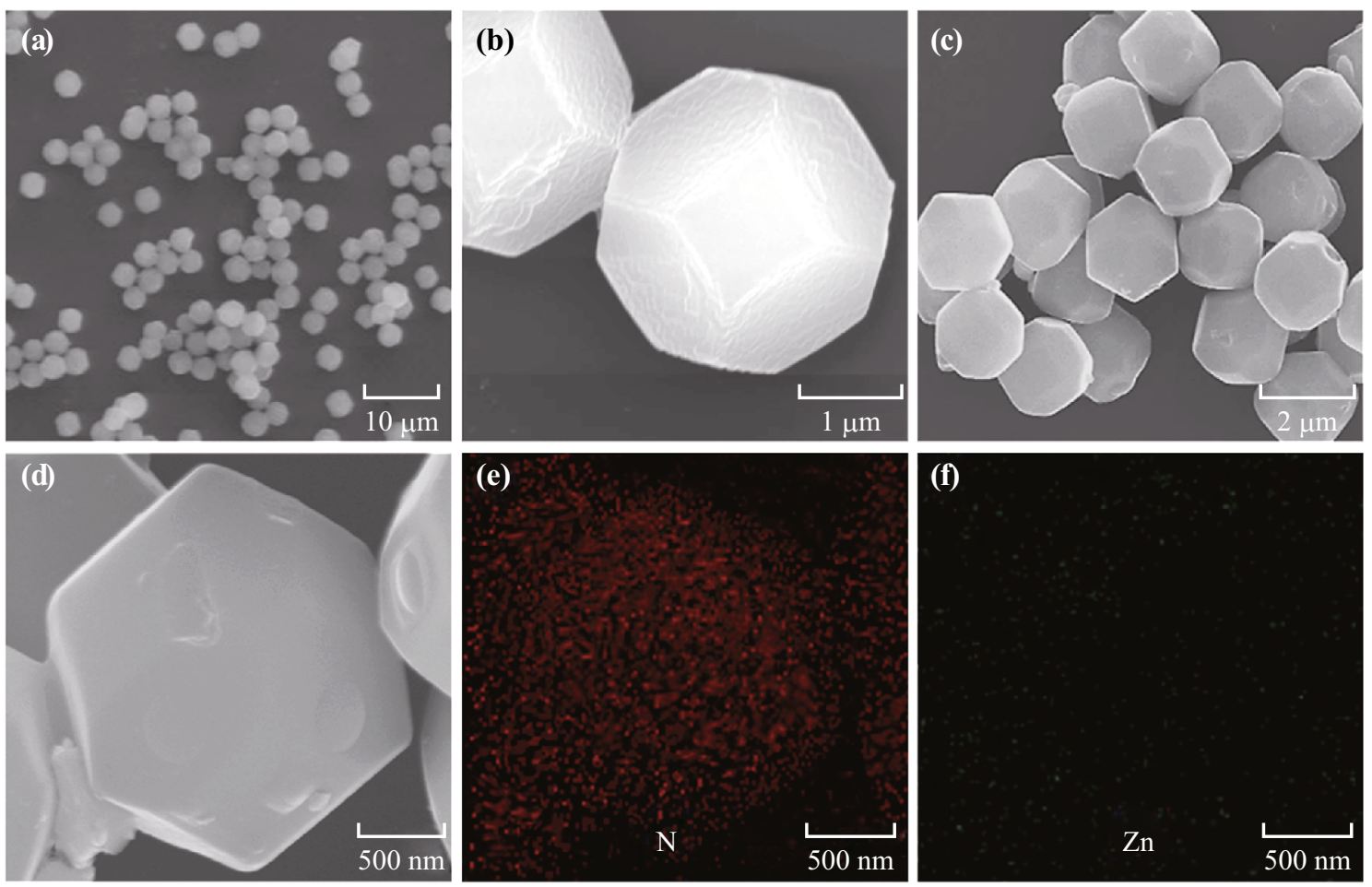

Fig. 1 SEM images of a, b ZIF-8, $\mathbf{c}, \mathbf{d}$ NPC and $\mathbf{e}, \mathbf{f}$ elemental mapping of NPC 
To get insight into the pore structure evolution before and after the incorporation of $\mathrm{Ag} \mathrm{NPs}$, the nitrogen absorption-desorption of NPC and Ag-NPC was measured. The Brunauer-Emmett-Teller (BET) surface of NPC and Ag-NPC was 844.715 and $270.174 \mathrm{~cm}^{3} \mathrm{~g}^{-1}$, respectively. Such a dramatic decrease could be the result of the encapsulation of Ag NPs into hierarchical pores of NPC. The $\mathrm{N}_{2}$ sorption isotherm of NPC (Fig. 2a) showed a pseudo-type IV isotherm and a hysteresis loop at a relative pressure $\left(\mathrm{P} / \mathrm{P}_{0}\right)$ of $0.4-0.95$, which suggests that it is a mesoporous structure, while the Ag-NPC showed a pseudotype I isotherm, which is consistent with the features of micropores. This indicated that the mesoporous structure of NPC turned into a microporous structure when Ag NPs were embedded, which can be supported by the pore diameter distribution diagram shown in Fig. $2 \mathrm{~b}$. The pore size of NPC was mainly centered at $2.17 \mathrm{~nm}$ (mesopores), while micropores were dominant in Ag-NPC. The pore

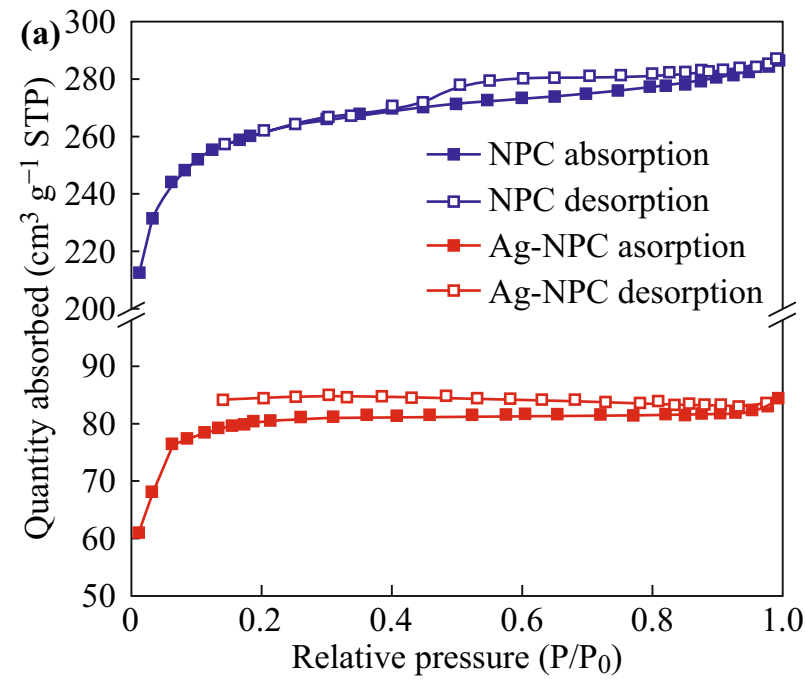

(b)

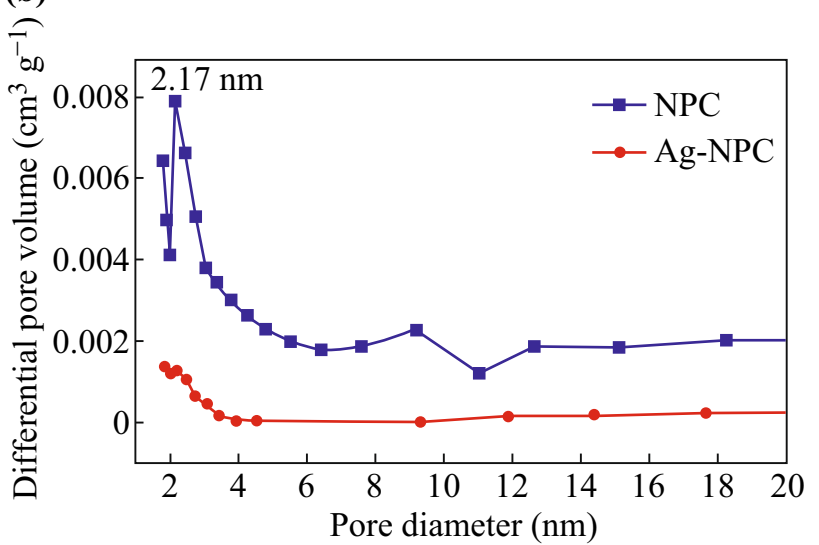

Fig. 2 a $\mathrm{N}_{2}$ absorption-desorption isotherms of NPC and Ag-NPC, and $\mathbf{b}$ pore size distribution of NPC and Ag-NPC quantities in Ag-NPC were much lower than that in NPC for each size, especially for $2.17 \mathrm{~nm}$.

Figue 3a shows XRD patterns of in situ synthesized ZIF8 and Ag-NPC composites. The XRD results of ZIF-8 agreed with the simulated pattern of ZIF-8. The peaks of $\mathrm{Ag}-\mathrm{NPC}$ at $38.2^{\circ}, 44.4^{\circ}, 64.6^{\circ}, 77.6^{\circ}$, and $81.8^{\circ}$ were typical of XRD patterns of metallic Ag. Notably, a weak and broad diffraction peak at $2 \theta$ with a value of approximately $25^{\circ}$ was observed, which was related to the (002) lattice plane of hexagonal graphitic carbon, indicating a low graphitic crystallinity of NPC matrix. This result could be corroborated by Raman spectroscopy of Ag-NPC as well. In Fig. 3b, the D-band represents the disordered graphitic crystallites of carbon and the inner defects of
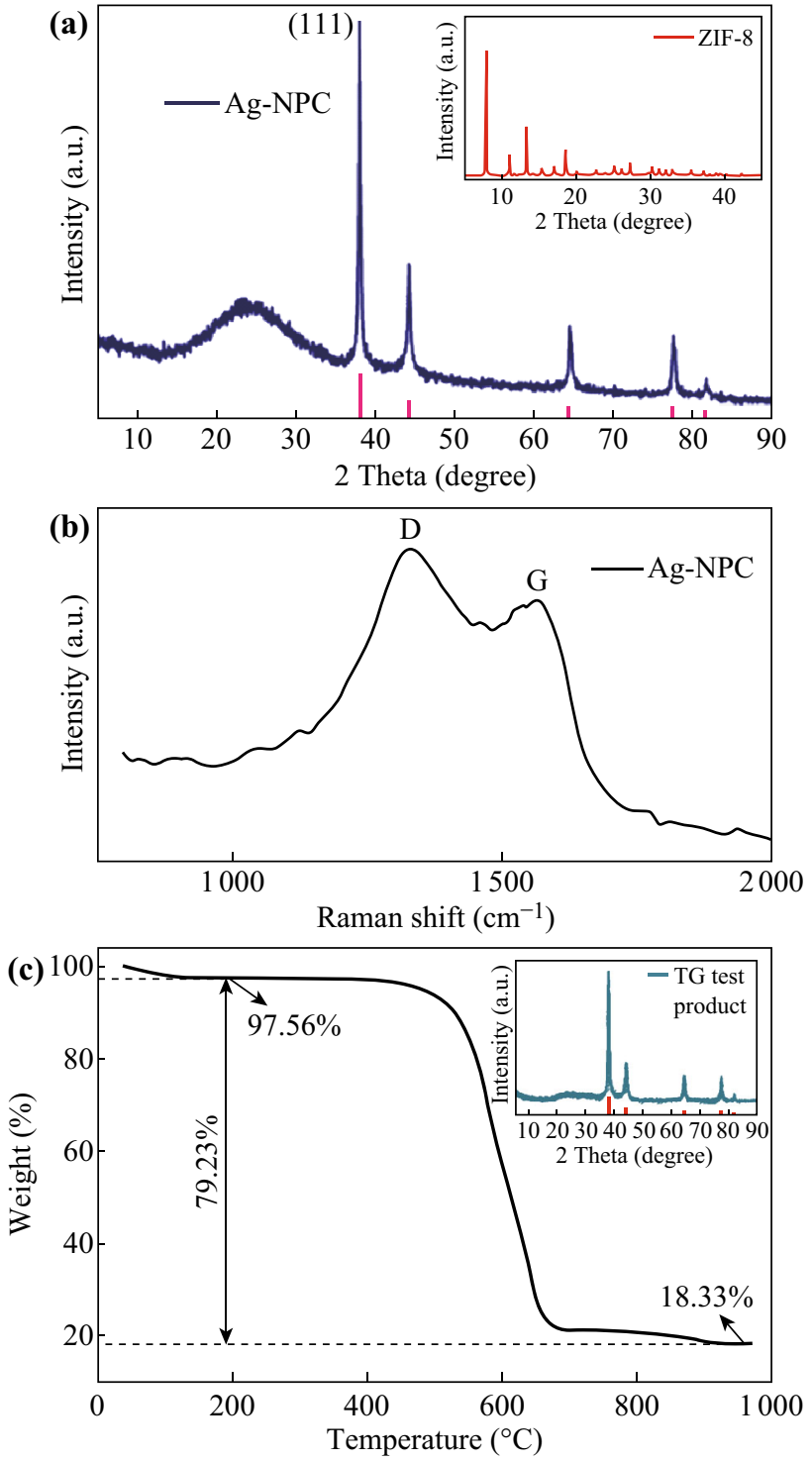

Fig. 3 a XRD patterns of the Ag-NPC composite and ZIF-8, b Raman spectra of Ag-NPC and c TGA analysis of Ag-NPC and XRD pattern of the product 
graphitic crystallites, while G-band represents the ordered section. The D/G ratio $\left(I_{\mathrm{D}} / I_{\mathrm{G}}\right)$ of Ag-NPC was 1.10 , indicating a highly disordered structure of NPC matrix.

To determine the content of Ag NPs, thermogravimetric analysis in air was carried out for the Ag-NPC. As shown in Fig. 3c, a slight weight loss was observed at temperatures lower than $150{ }^{\circ} \mathrm{C}$, which was probably caused by the evaporation of absorbed water. Then, the curve was flat until $500{ }^{\circ} \mathrm{C}$, at which the nitrogen-doped carbon began to decompose. When the temperature increased over $900{ }^{\circ} \mathrm{C}$, the curve became flat again, indicating complete decomposition of the carbon matrix. The resulting product of TGA proved to be metallic silver, confirmed by means of XRD. That is, the weight percentage of Ag NPs in Ag-NPC was $18.79 \%$.

The morphology of Ag-NPC was determined from SEM images. As shown in Fig. 4a, Ag-NPC kept the size and morphology of NPC particles. From the high-magnified SEM image shown in Fig. 4b, it was clear that many nanoparticles were attached to the surface of NPC, which was different from the smooth surface of NPC (Fig. 1d). The elemental mapping in Fig. $4 \mathrm{c}$ implied that Ag NPs were uniformly encapsulated in NPC and evenly attached to its surface. A more detailed investigation was performed using HRTEM (Fig. 4d). The particle size of Ag NPs in Fig. 4e ranged from 8 to $20 \mathrm{~nm}$. The lattice distance in the related high-resolution TEM image was about $0.23 \mathrm{~nm}$
(Fig. 4f), which was consistent with the interplanar distance of d (111) in the Ag crystal.

The chemical nature of the Ag-NPC can be analyzed by XPS characterization, as shown in Fig. 5a. The wide-survey scan XPS spectrum corroborated the presence of $\mathrm{C}, \mathrm{N}$ and Ag elements in Ag-NPC. The high-resolution Ag 3d spectrum (Fig. 5b) showed the core level binding energies at 368.3 and $374.3 \mathrm{eV}$ that corresponded to $\mathrm{Ag} 3 \mathrm{~d}_{5 / 2}$ and $\mathrm{Ag} 3 \mathrm{~d}_{3 / 2}$, respectively. The spin energy separation of $6.0 \mathrm{eV}$ showed evidence of the metallic nature of silver nanocrystals [34]. The $\mathrm{N}$ 1s spectrum (Fig. 5c) can be decomposed into peaks that matched different chemical states, pyridinic $\mathrm{N}(398.57 \mathrm{eV})$, pyrrolic $\mathrm{N}(399.89 \mathrm{eV})$ and quaternary $\mathrm{N}(401.01 \mathrm{eV})$ of nitrogen. Further quantitative analysis of the $\mathrm{N} \mathrm{1s}$ spectrum showed that the majority of $\mathrm{N}$ in Ag-NPC existed in the form of pyridinic $\mathrm{N}$ $(\approx 53.2 \mathrm{wt} \%$ ), which has a lone pair of electrons that could facilitate the electron transfer [35].

According to previous discussion and analysis, the schematic diagram of the formation process of Ag-NPC is presented in Fig. 6 . ZIF-8 was pyrolyzed at $800{ }^{\circ} \mathrm{C}$ followed by acid pickling with $\mathrm{HCl}$ to produce $\mathrm{N}$-doped porous carbon. Then, the $\mathrm{N}$-doped porous carbon was immersed into an $\mathrm{AgNO}_{3}$ solution and stirred intensely to allow the $\mathrm{Ag}^{+}$to diffuse into the pores inside the NPC and tightly adhere to the pore surface due to electrostatic interaction. Through heat treatment at $100{ }^{\circ} \mathrm{C}$ under the
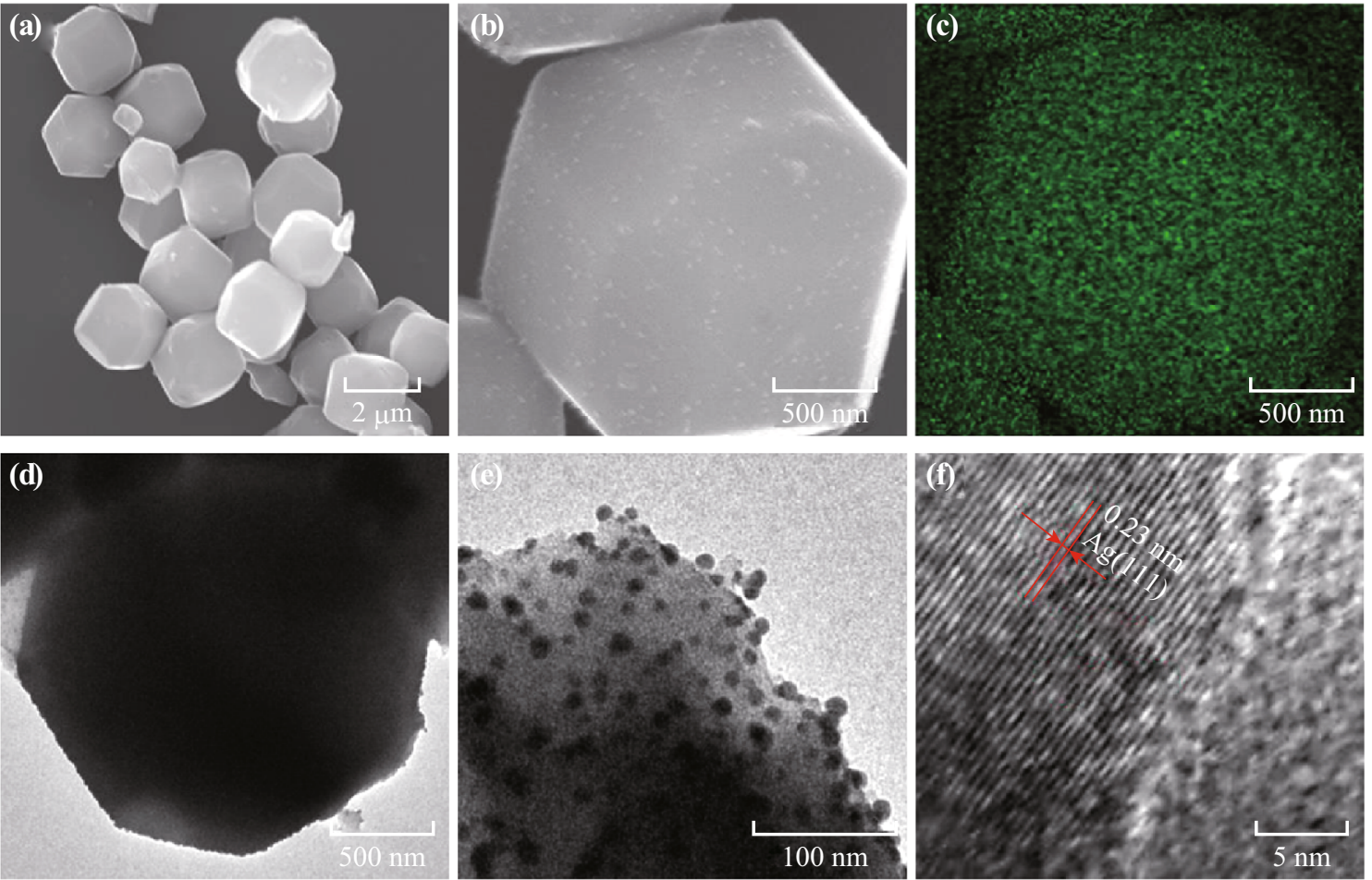

Fig. 4 a, b SEM micrograph of Ag-NPC, $\mathbf{c}$ elemental mapping of Ag-NPC, d, e TEM image of Ag-NPC and $\mathbf{f}$ HRTEM of Ag NPs on the surface of NPC 

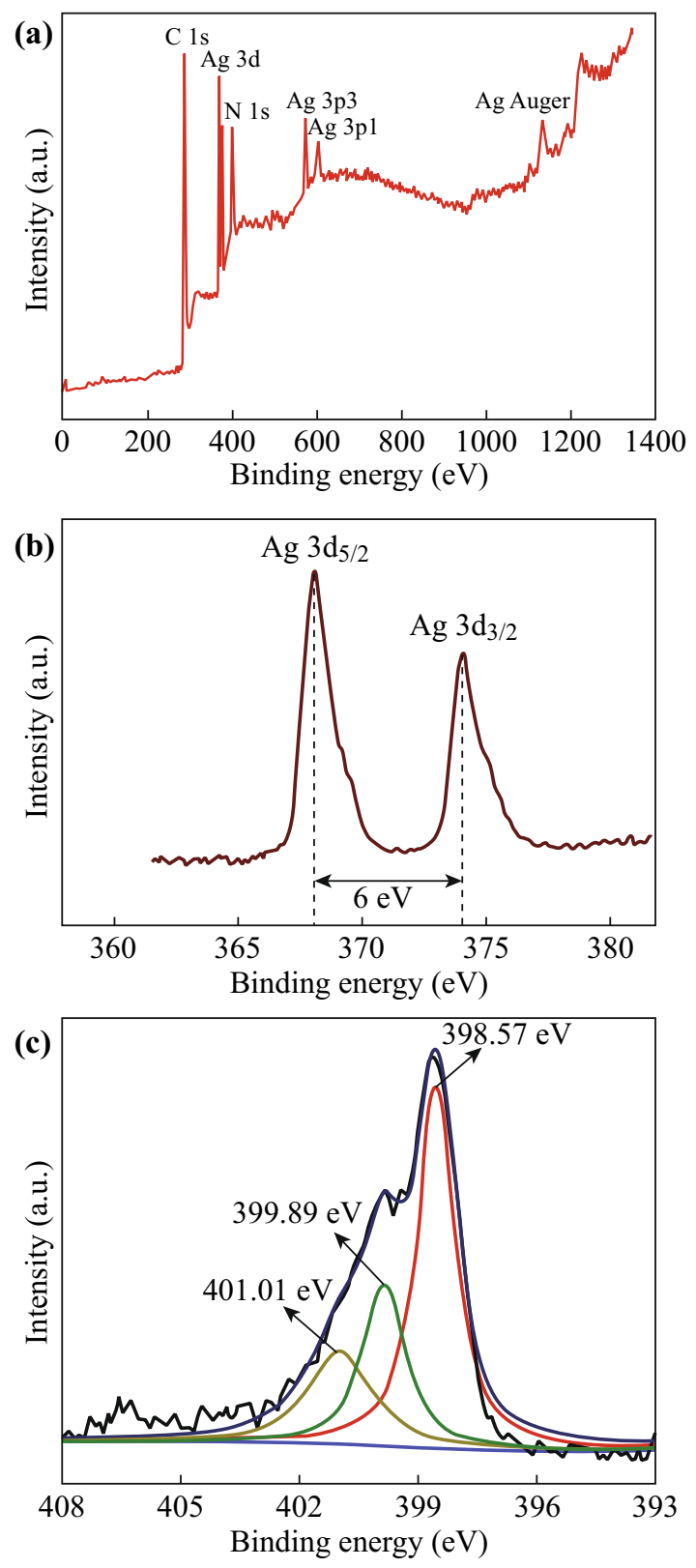

Fig. 5 a XPS full spectra of Ag-NPC composite. The high-resolution XPS spectra of $\mathbf{b} \mathrm{Ag} 3 \mathrm{~d}$ and $\mathbf{c} \mathrm{N} 1 \mathrm{~s}$

argon flow, $\mathrm{AgNO}_{3}$ was decomposed into metallic silver. Thus, Ag NPs were uniformly embedded inside the carbon matrix and deposited onto the outer surface of the NPC microparticles.

The electrochemical properties of the Ag-NPC and NPC were measured. To investigate the electrode reactions of NPC and Ag-NPC during the $\mathrm{Li}^{+}$insertion/extraction process, $\mathrm{CV}$ characterization was performed at a scan rate of $0.1 \mathrm{mV} \mathrm{s}^{-1}$ between 0.01 and $3 \mathrm{~V}$. Figure $7 \mathrm{a}$ shows the CV profile of the NPC. In the first cycle, a broad cathodic peak was observed at $0.2-1.0 \mathrm{~V}$, which was assigned to the formation of the solid electrolyte interface (SEI) films. The sharp peak that appeared near $0 \mathrm{~V}$ resulted from the $\mathrm{Li}^{+}$ insertion into the NPC. In addition, a relatively weak cathodic peak from $1.0 \mathrm{~V}$ to $1.4 \mathrm{~V}$ could be due to $\mathrm{Li}^{+}$ binding with $\mathrm{N}$ atoms on the surface of its internal pores [35, 36]. Notably, for the CV profile of the Ag-NPC (Fig. 7b), the redox peak related to the formation of $\mathrm{Li}_{\mathrm{x}} \mathrm{N}$ appeared between 0.7 and $0.9 \mathrm{~V}$, showing a small shift compared to that of the NPC. This phenomenon was because the reaction of $\mathrm{Li}^{+}$with nitrogen was catalyzed by the Ag NPs [37]. Furthermore, a new peak appeared at 1.3-1.7 V after the embedding of Ag NPs. This resulted from the alloying reaction of $\mathrm{Li}^{+}$with $\mathrm{Ag}$ NPs. Correspondingly, in the anodic scan, the peaks related to the dealloying process of $\mathrm{Li}-\mathrm{Ag}$ can be observed at 0.35 and $0.12 \mathrm{~V}$. The electrochemical reaction was described as follows [38],

$x \mathrm{Li}^{+}+x \mathrm{e}^{-}+\mathrm{Ag} \leftrightarrow \mathrm{Li}_{x} \mathrm{Ag}$

We found that the third cycle almost overlapped with the second cycle in the $\mathrm{CV}$ profile of Ag-NPC in contrast to $\mathrm{NPC}$, proving that the Ag-NPC displayed a better cycling stability than the NPC did.

Figure 8a shows the analysis of electrochemical impedance spectroscopy (EIS) measurement of NPC and AgNPC. Each Nyquist plot showed a semicircle in the highto-medium frequency region, which was related to the charge-transfer process [39]. The charge-transfer resistance of the Ag-NPC electrode was $50 \Omega$, much smaller than that of the NPC electrode $(100 \Omega)$. This demonstrated that the encapsulation of Ag NPs efficiently enhanced the chargetransfer process due to improvement in the electrical conductivity. The linear tails in the Warburg region corresponded to the mass transfer of $\mathrm{Li}^{+}$inside the anode material. The Ag-NPC exhibited a larger slope than the bare NPC, indicating a higher $\mathrm{Li}^{+}$migration velocity with the incorporation of Ag NPs. Therefore, it can be concluded that Ag NPs can promote both electron transfer and lithium-ion transfer, which has a significant impact on electrochemical performance [40].

Figure $8 \mathrm{~b}$ displays the galvanostatic charge-discharge profile of NPC and Ag-NPC for first, second and third cycles at a current density of $100 \mathrm{~mA} \mathrm{~g}^{-1}$. In the initial discharge curve of NPC, a broad plateau appeared near $0.75 \mathrm{~V}$, which was usually related to the formation of the SEI films. The plateau for the Ag-NPC around $0.75 \mathrm{~V}$ was much narrower than that for the NPC, signaling that smaller areas of SEI films were formed, resulting in less capacity loss. However, no plateau could be detected in the charge curves of the NPC, implying continuous $\mathrm{Li}^{+}$ extraction from the NPC. It was noteworthy that for the charge-discharge profile of the Ag-NPC, several small plateaus can be observed apart from the one at $0.75 \mathrm{~V}$. In the discharge curve, plateaus were at $0.076,0.10$, and 


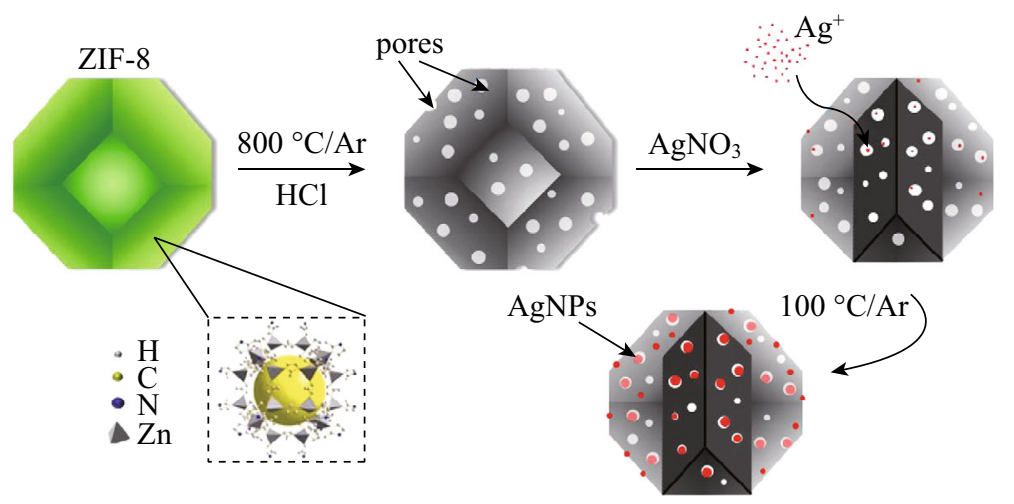

Fig. 6 Schematic diagram of Ag-NPC formation process
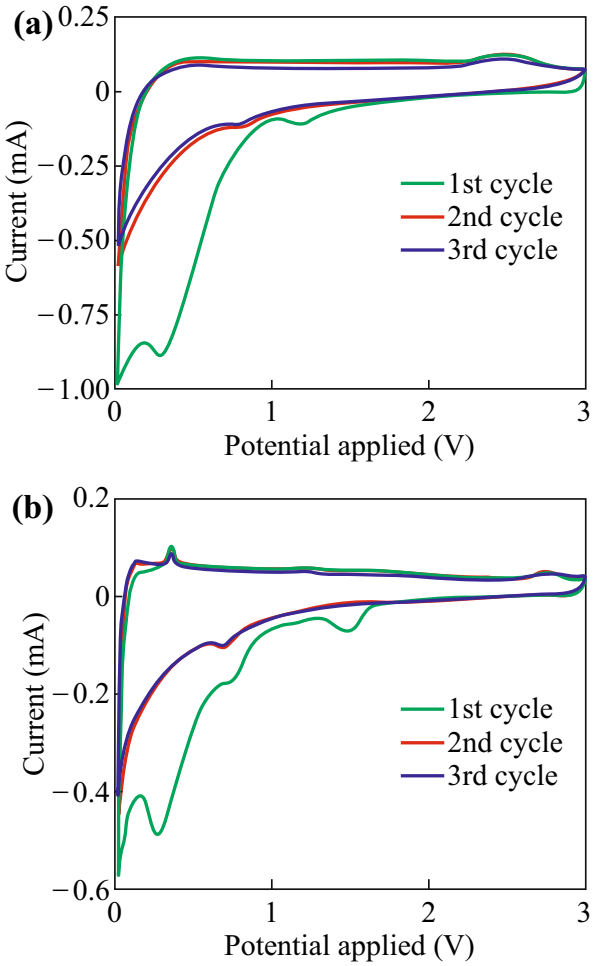

Fig. 7 Cyclic voltammogram of a NPC and b Ag-NPC electrode obtained at $0.1 \mathrm{mV} \mathrm{s}^{-1}$ in the potential window $0.01-3 \mathrm{~V}$

$0.2 \mathrm{~V}$, respectively. The charge curve presented several small plateaus within the range of $0.1-0.3 \mathrm{~V}$. The step-like curves correlated with the complex alloying and dealloying processes of $\mathrm{Ag}$ NPs with $\mathrm{Li}^{+}$.

The cycling performance of NPC and Ag-NPC was investigated at a current density of $0.1 \mathrm{~A} \mathrm{~g}^{-1}$ (Fig. 8c). The initial discharge capacity for NPC was very high (2226.64 $\mathrm{mAh} \mathrm{g}^{-1}$ ), but a dramatic capacity loss occurred in the first 25 cycles, leading to a low initial coulombic efficiency $(43.41 \%)$ as well as poor cycling performance. After the encapsulation of Ag NPs, the initial discharge capacity dropped to $1374.64 \mathrm{mAh} \mathrm{g}^{-1}$ though its initial coulombic efficiency improved to $64.33 \%$. In the following cycles, the coulombic efficiency quickly increased above 95\%, which meant a better cycling performance of AgNPC than NPC. The reason was that Ag-NPC had a much smaller specific surface area than NPC (270.174 vs $844.715 \mathrm{~cm}^{3} \mathrm{~g}^{-1}$ ), so smaller areas of SEI films were formed during cycles. Because the formation of SEI films contributed significantly to the initial capacity [4], the NPC with a much larger area showed a very high initial discharge capacity. However, the reaction of the formation of SEI was always irreversible, so NPC suffered a dramatic capacity loss in the second cycle. In addition, Ag NPs could improve the quality of SEI films, leading to a better cycling performance. Further, after 200 cycles, the reversible capacity for Ag-NPC remained at $852 \mathrm{mAh} \mathrm{g}^{-1}$, which was $96.4 \%$ of the discharge capacity in the second cycle, while that for NPC was only $501.6 \mathrm{mAh} \mathrm{g}^{-1}$. In other words, the Ag NPs can not only efficiently improve the reversible discharge capacity, but also enhance the cycling stability of the NPC. This remarkable improvement was attributed to the synergistic effect of Ag NPs and the carbon matrix. The theoretical capacity of metallic Ag was $993 \mathrm{mAh} \mathrm{g}^{-1}$ (for $\mathrm{Li}_{4} \mathrm{Ag}$ ), although it suffered from severe volume expansion and agglomeration, leading to poor cycling performance. In the Ag-NPC, the NPC acted as a robust matrix for Ag NPs, hence, alleviating the volumetric expansion and preventing particles from aggregating. Moreover, the encapsulation of Ag NPs reduced the surface contact of the NPC with the electrolytes, preventing the formation of unstable SEI films and improving the quality of SEI films and thus enhancing the cycling performance [9]. Further, the excellent electrical conductivity of Ag NPs accelerated electron transfer and lithium-ion transfer, thus boosting the electrochemical performance.

It is noteworthy that the calculated theoretical capacity for Ag-NPC was found to be $488 \mathrm{mAh} \mathrm{g}^{-1}$ based on $\mathrm{AgLi}_{4}$ and $\mathrm{C}_{6} \mathrm{Li}$, which was much lower than the measured capacity of $852 \mathrm{mAh} \mathrm{g}^{-1}$. This phenomenon had also been reported by previous papers [41-43]. This was contributed 

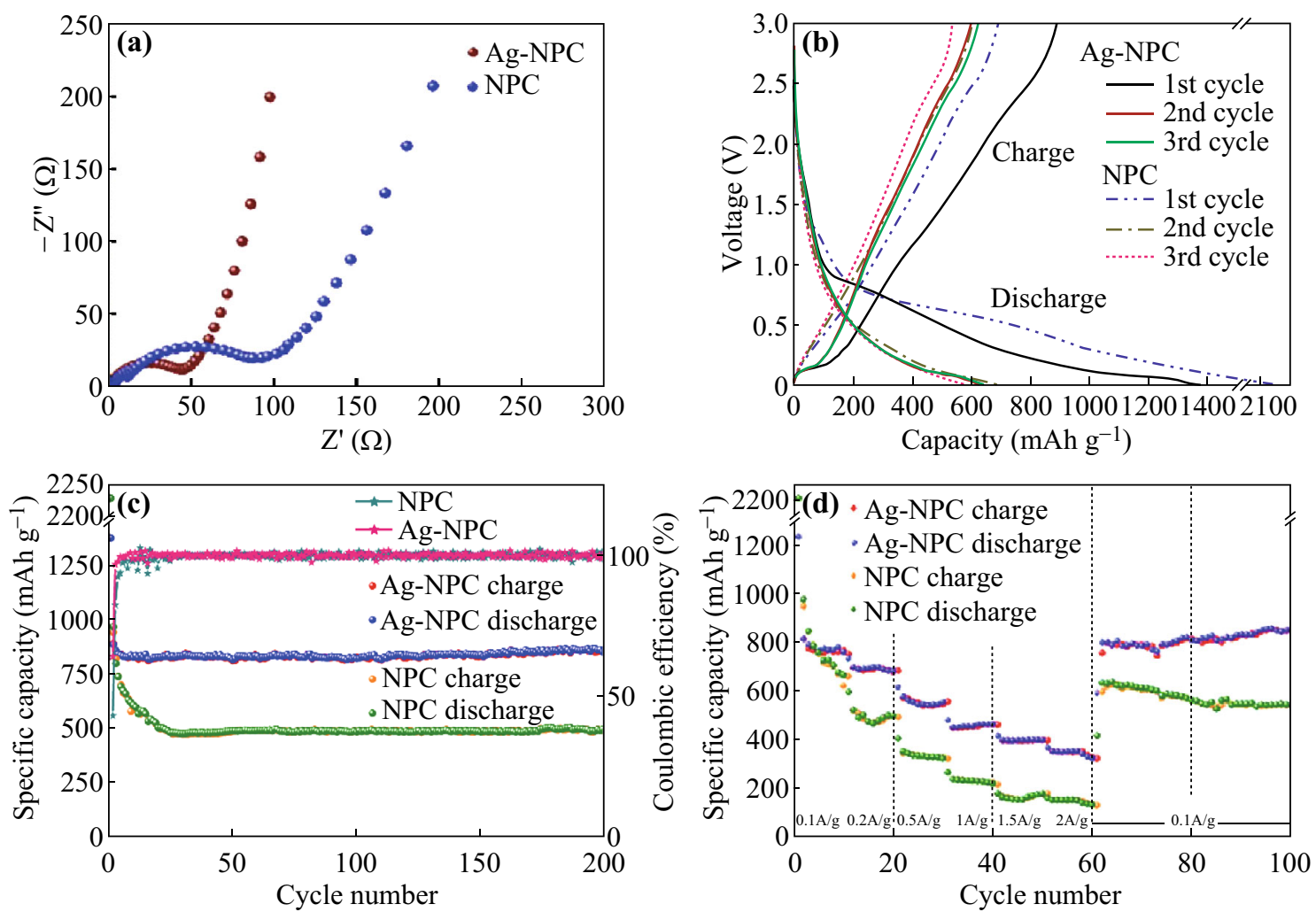

Fig. 8 a Electrochemical impedance spectra, b the galvanostatic discharge-charge profiles at $100 \mathrm{~mA} \mathrm{~g}^{-1}$, and $\mathbf{c}$ the cycling properties and d rate capabilities of NPC and Ag-NPC

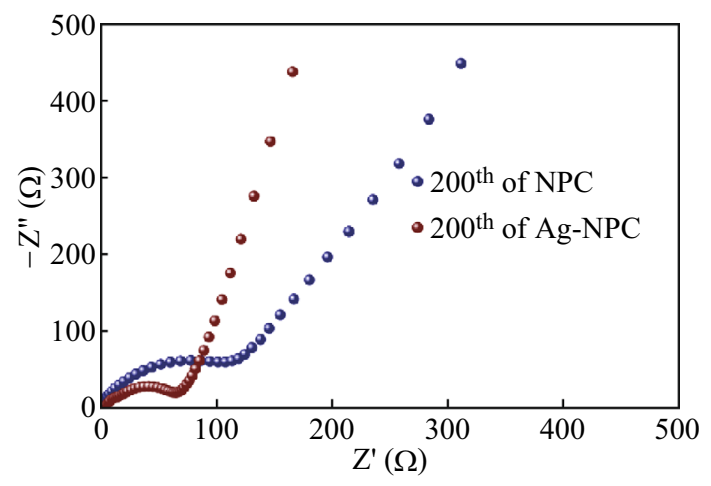

Fig. 9 Electrochemical impedance spectra in the $200^{\text {th }}$ cycle of NPC and Ag-NPC

to the highly disordered structure of carbon matrices, high nitrogen content, as well as the improved conductivity of Ag NPs. The carbon matrix was highly disordered according to the broad peak in the XRD pattern and the Raman spectroscopy results of Ag-NPC discussed above. The defects in the carbon matrix promoted $\mathrm{Li}^{+}$diffusion and intercalation into carbon, thereby improving its reversible capacity [42]. Besides, the high nitrogen content (17 wt $\%$ ) can efficiently facilitate electron transfer, thus increasing the capacity. Furthermore, the superior conductivity of Ag NPs raised the electrical conductivity of the overall Ag-NPC composite and boosted the reversible capacity.

The rate performance for NPC and Ag-NPC was analyzed at different current rates as shown in Fig. 8d. The reversible capacity for both materials gradually decreased with the current rate. The Ag-NPC delivered a much higher discharge capacity than the NPC did at each current density level. The average discharge capacities of Ag-NPC were 777.69, $696.08,557.30,459.47,397.98$, and $344.40 \mathrm{mAh} \mathrm{g}^{-1}$ for the increasing current values $0.1,0.2,0.5,1.0,1.5$, and $2.0 \mathrm{~A} \mathrm{~g}^{-1}$, respectively. When the current density was lowered back to $0.1 \mathrm{~A} \mathrm{~g}^{-1}$, its discharge capacity recovered to $851.02 \mathrm{mAh} \mathrm{g}^{-1}$ at the 100th cycle. The rate behavior demonstrated its superior rate capacity, which could be attributed to the rigid porous structure of the carbon matrix.

To further understand the improved cycling performance, we performed EIS measurements after 200 cycles at a current density of $100 \mathrm{~mA} \mathrm{~g}^{-1}$ (Fig. 9). Compared to the EIS results in the first cycle shown in Fig. 8a, both NPC and Ag-NPC displayed a slight increase in charge-transfer resistance after 200 cycles, indicating that they possessed a robust structure, which was mainly attributed to the rigid structure of the carbon matrix that it inherited from the 

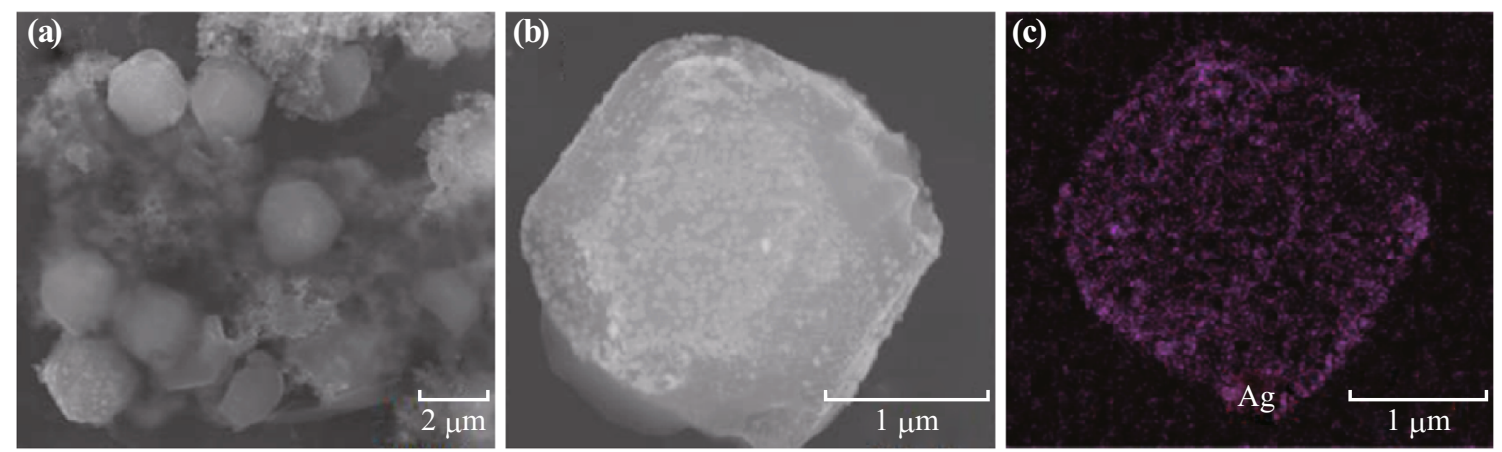

Fig. 10 a, b SEM micrographs and c elemental mapping of Ag-NPC after 200 cycles at $100 \mathrm{~mA} \mathrm{~g}^{-1}$

ZIF-8 precursor. The structural stability of the Ag-NPC could be demonstrated visually by SEM. In Fig. 10a, neither agglomeration nor volume expansion to a carbon matrix can be observed. Meanwhile, the original morphology of Ag-NPC, rhombic dodecahedron, was well maintained after 200 cycles at $0.1 \mathrm{~A} \mathrm{~g}^{-1}$ (Fig. 10b).It was noteworthy that Ag NPs were still tightly attached to the surface of the NPC, yet were highly dispersed (Fig. 10c) after 200 cycles, despite the volume expansion of the Ag NPs. This suggests the excellent structural stability of the Ag-NPC composite.

\section{Conclusions}

In summary, a novel Ag-NPC composite with uniformly embedded Ag NPs was prepared through the facile hydrothermal method. The Ag-NPC composite showed remarkable enhancement in the reversible capacity (852 $\mathrm{mAh} \mathrm{g}^{-1}$ after 200 cycles) as well as cycling performance, compared to NPC without incorporated Ag NPs. This was attributed to the synergistic effect of Ag NPs and $\mathrm{N}$-doped porous carbon. The $\mathrm{N}$-doped porous carbon acted as a robust matrix for Ag NPs, which could alleviate the volumetric expansion and prevent particle aggregation. In return, the Ag NPs that exhibited a relatively high specific capacity as well as superior conductivity could efficiently raise its reversible capacity and enhance cycling performance by improving the quality of SEI films. This research demonstrated that the NPC derived from ZIF-8 was an excellent matrix for nanoparticles in anode materials for lithium-ion batteries. By providing a relatively rigid structure and avoiding particle aggregation, the encapsulated nanoparticles could maximize the reversible capacities.

Acknowledgements This work was supported by the Scientific and Technological Innovation Platform of Fujian Province (2006L2003).
Open Access This article is distributed under the terms of the Creative Commons Attribution 4.0 International License (http://crea tivecommons.org/licenses/by/4.0/), which permits unrestricted use, distribution, and reproduction in any medium, provided you give appropriate credit to the original author(s) and the source, provide a link to the Creative Commons license, and indicate if changes were made.

\section{References}

1. Q.S. Xie, Y.T. Ma, D.Q. Zeng, X.Q. Zhang, L.S. Wang, G.H. Yue, D.L. Peng, Hierarchical $\mathrm{ZnO}-\mathrm{Ag}-\mathrm{C}$ composite porous microspheres with superior electrochemical properties as anode materials for lithium ion batteries. ACS Appl. Mater. Interfaces 6(22), 19895-19904 (2014). doi:10.1021/am505352p

2. X. Zhang, J. Ma, K. Chen, Impact of morphology of conductive agent and anode material on lithium storage properties. NanoMicro Lett. 7(4), 360-367 (2015). doi:10.1007/s40820-015-00517

3. Y.-M. Chiang, Building a better battery. Science 330(6010), 1485-1486 (2010). doi:10.1126/science.1198591

4. Y. Han, P. Qi, S. Li, X. Feng, J. Zhou, H. Li, S. Su, X. Li, B. Wang, A novel anode material derived from organic-coated ZIF8 nanocomposites with high performance in lithium ion batteries. Chem. Commun. 50(59), 8057-8060 (2014). doi:10.1039/ $\mathrm{c} 4 \mathrm{cc} 02691 \mathrm{~h}$

5. Z.S. Wu, W.C. Ren, L. Wen, L.B. Gao, J.P. Zhao, Z.P. Chen, G.M. Zhou, F. Li, H.M. Cheng, Graphene anchored with $\mathrm{Co}_{3} \mathrm{O}_{4}$ nanoparticles as anode of lithium ion batteries with enhanced reversible capacity and cyclic performance. ACS Nano 4(6), 3187-3194 (2010). doi:10.1021/nn100740x

6. X. Liu, C. Zhao, H. Zhang, Q. Shen, Facile synthesis of porous $\mathrm{ZnMnO}_{3}$ spherulites with a high lithium storage capability. Electrochim. Acta 151, 56-62 (2015). doi:10.1016/j.electacta. 2014.11.020

7. S. Zhao, Y. Wang, R. Liu, Y. Yu, S. Wei, F. Yu, Q. Shen, Fullmolar-ratio synthesis and enhanced lithium storage properties of $\mathrm{CoxFe} 1-\mathrm{xCO}_{3}$ composites with an integrated lattice structure and an atomic-scale synergistic effect. J. Mater. Chem. A 3(33), 17181-17189 (2015). doi:10.1039/c5ta03785a

8. F. Feng, W. Kang, F. Yu, H. Zhang, Q. Shen, High-rate lithium storage capability of cupric-cobaltous oxalate induced by unavoidable crystal water and functionalized graphene oxide. J. Power Sources 282, 109-117 (2015). doi:10.1016/j.jpowsour. 2015.02.043 
9. F.H. Du, K.X. Wang, W. Fu, P.F. Gao, J.F. Wang, J. Yang, J.S. Chen, A graphene-wrapped silver-porous silicon composite with enhanced electrochemical performance for lithium-ion batteries. J. Mater. Chem. A 1(43), 13648-13654 (2013). doi:10.1039/ c3ta13092d

10. J.X. Song, S.R. Chen, M.J. Zhou, T. Xu, D.P. Lv et al., Microsized silicon-carbon composites composed of carbon-coated sub$10 \mathrm{~nm} \mathrm{Si} \mathrm{primary} \mathrm{particles} \mathrm{as} \mathrm{high-performance} \mathrm{anode} \mathrm{materials}$ for lithium-ion batteries. J. Mater. Chem. A 2(5), 1257-1262 (2014). doi:10.1039/c3ta14100d

11. C.D. Wang, Y.S. Chui, R.G. Ma, T.L. Wong, J.G. Ren, Q.H. Wu, X.F. Chen, W.J. Zhang, A three-dimensional graphene scaffold supported thin film silicon anode for lithium-ion batteries. J. Mater. Chem. A 1(35), 10092-10098 (2013). doi:10.1039/ c3ta11740e

12. K. Shiva, K. Jayaramulu, H.B. Rajendra, T. Maji, A.J. Bhattacharyya, In-situ stabilization of tin nanoparticles in porous carbon matrix derived from metal organic framework: high capacity and high rate capability anodes for lithium-ion batteries. Z. Anorg. Allg. Chem. 640(6), 1115-1118 (2014). doi:10.1002/ zaac. 201300621

13. Y.X. Wang, Y.G. Lim, M.S. Park, S.L. Chou, J.H. Kim, H.K. Liu, S.X. Dou, Y.J. Kim, Ultrafine $\mathrm{SnO}_{2}$ nanoparticle loading onto reduced graphene oxide as anodes for sodium-ion batteries with superior rate and cycling performances. J. Mater. Chem. A 2(2), 529-534 (2014). doi:10.1039/c3ta13592f

14. J.F. Yin, H.Q. Cao, Z.F. Zhou, J.X. Zhang, M.Z. Qu, SnS $\mathrm{S}_{2} @$ reduced graphene oxide nanocomposites as anode materials with high capacity for rechargeable lithium ion batteries. J. Mater. Chem. 22(45), 23963-23970 (2012). doi:10.1039/c2jm35137d

15. A. Shilpa, Sharma, Enhanced electrochemical performance of electrospun ag/hollow glassy carbon nanofibers as free-standing li-ion battery anode. Electrochim. Acta 176, 1266-1271 (2015). doi:10.1016/j.electacta.2015.07.093

16. G. Taillades, J. Sarradin, Silver: high performance anode for thin film lithium ion batteries. J. Power Sources 125(2), 199-205 (2004). doi:10.1016/j.jpowsour.2003.07.004

17. S. Li, J.G. Huang, A nanofibrous silver-nanoparticle/titania/carbon composite as an anode material for lithium ion batteries. J. Mater. Chem. A 3(8), 4354-4360 (2015). doi:10.1039/ c4ta06562j

18. Y. Dai, S.D. Cai, W.J. Yang, L. Gao, W.P. Tang, J.Y. Xie, J. Zhi, X.M. Ju, Fabrication of self-binding noble metal/flexible graphene composite paper. Carbon 50(12), 4648-4654 (2012). doi:10.1016/j.carbon.2012.05.053

19. R. Chen, S.Z. Zhao, G.Y. Han, J.H. Dong, Fabrication of the silver/polypyrrole/polyacrylonitrile composite nanofibrous mats. Mater. Lett. 62(24), 4031-4034 (2008). doi:10.1016/j.matlet. 2008.05.054

20. J.T. Yin, M. Wada, Y. Kitano, S. Tanase, O. Kajita, T. Sakai, Nanostructured Ag-Fe-Sn/carbon nanotubes composites as anode materials for advanced lithium-ion batteries. J. Electrochem. Soc. 152(7), A1341-A1346 (2005). doi:10.1149/1.1921727

21. C.T. Hsieh, C.Y. Lin, Y.F. Chen, J.S. Lin, H. Teng, Silver nanorods attached to graphene sheets as anode materials for lithium-ion batteries. Carbon 62, 109-116 (2013). doi:10.1016/j. carbon.2013.06.002

22. Z.Q. Li, L.W. Yin, Sandwich-like reduced graphene oxide wrapped MOF-derived $\mathrm{ZnCo}_{2} \mathrm{O}_{4}-\mathrm{ZnO}-\mathrm{C}$ on nickel foam as anodes for high performance lithium ion batteries. J. Mater. Chem. A 3(43), 21569-21577 (2015). doi:10.1039/c5ta05733g

23. C. Li, T. Chen, W. Xu, X. Lou, L. Pan, Q. Chen, B. Hu, Mesoporous nanostructured $\mathrm{Co}_{3} \mathrm{O}_{4}$ derived from MOF template: a high-performance anode material for lithium-ion batteries. J. Mater. Chem. A 3(10), 5585-5591 (2015). doi:10.1039/ c4ta06914e
24. J.J. Ma, H.J. Wang, X. Yang, Y.Q. Chai, R. Yuan, Porous carboncoated $\mathrm{CuCO}_{2} \mathrm{O}_{4}$ concave polyhedrons derived from metal-organic frameworks as anodes for lithium-ion batteries. J. Mater. Chem. A 3(22), 12038-12043 (2015). doi:10.1039/c5ta00890e

25. L. Hu, Q.W. Chen, Hollow/porous nanostructures derived from nanoscale metal-organic frameworks towards high performance anodes for lithium-ion batteries. Nanoscale 6(3), 1236-1257 (2014). doi:10.1039/c3nr05192g

26. F. Zheng, Y. Yang, Q. Chen, High lithium anodic performance of highly nitrogen-doped porous carbon prepared from a metal-organic framework. Nat. Commun. 5, 5261 (2014). doi:10.1038/ ncomms6261

27. H.-G. Wang, Y. Wang, Y. Li, Y. Wan, Q. Duan, Exceptional electrochemical performance of nitrogen-doped porous carbon for lithium storage. Carbon 82, 116-123 (2015). doi:10.1016/j. carbon.2014.10.041

28. P. Li, M. Cui, M. Zhang, A. Guo, Y. Sun, H.-G. Wang, Y. Li, Q. Duan, Facile fabrication of $\mathrm{Co}_{3} \mathrm{O}_{4} /$ nitrogen-doped graphene hybrid materials as high performance anode materials for lithium ion batteries. CrystEngComm 18(19), 3383-3388 (2016). doi:10. 1039/c6ce00462h

29. C. Jiang, C. Yuan, P. Li, H.-G. Wang, Y. Li, Q. Duan, Nitrogendoped porous graphene with surface decorated $\mathrm{MnO}_{2}$ nanowires as a high-performance anode material for lithium-ion batteries. J. Mater. Chem. A 4(19), 7251-7256 (2016). doi:10.1039/ c5ta10711c

30. Y.H. Song, L. Zuo, S.H. Chen, J.F. Wu, H.Q. Hou, L. Wang, Porous nano-si/carbon derived from zeolitic imidazolate frameworks@nano-si as anode materials for lithium-ion batteries. Electrochim. Acta 173, 588-594 (2015). doi:10.1016/j.electacta. 2015.05.111

31. Z. Xie, Z. He, X. Feng, W. Xu, X. Cui et al., Hierarchical sandwich-like structure of ultrafine n-rich porous carbon nanospheres grown on graphene sheets as superior lithium-ion battery anodes. ACS Appl. Mater. Interfaces 8(16), 10324-10333 (2016). doi:10.1021/acsami.6b01430

32. J. Cravillon, R. Nayuk, S. Springer, A. Feldhoff, K. Huber, M. Wiebcke, Controlling zeolitic imidazolate framework nano- and microcrystal formation: insight into crystal growth by time-resolved in situ static light scattering. Chem. Mater. 23(8), 2130-2141 (2011). doi:10.1021/cm103571y

33. S.X. Hu, Y.L. Hsieh, Synthesis of surface bound silver nanoparticles on cellulose fibers using lignin as multi-functional agent. Carbohydr. Polym. 131, 134-141 (2015). doi:10.1016/j. carbpol.2015.05.060

34. D. Zhang, Y. Li, M. Yan, Y.Z. Jiang, $\mathrm{Fe}_{2} \mathrm{O}_{3}-\mathrm{Ag}$ porous film anodes for ultrahigh-rate lithium-ion batteries. Chemelectrochem 1(7), 1155-1160 (2014). doi:10.1002/celc.201402045

35. K. Huo, W. An, J. Fu, B. Gao, L. Wang, X. Peng, G.J. Cheng, P.K. Chu, Mesoporous nitrogen-doped carbon hollow spheres as high-performance anodes for lithium-ion batteries. J. Power Sources 324, 233-238 (2016). doi:10.1016/j.jpowsour.2016.05. 084

36. K.L. Zhang, X.N. Li, J.W. Liang, Y.C. Zhu, L. Hu et al., Nitrogen-doped porous interconnected double-shelled hollow carbon spheres with high capacity for lithium ion batteries and sodium ion batteries. Electrochim. Acta 155, 174-182 (2015). doi:10.1016/j.electacta.2014.12.108

37. X.L. He, D. Hubble, R. Calzada, A. Ashtamkar, D. Bhatia, S. Cartagena, P. Mukherjee, H. Liang, A silver-nanoparticle-catalyzed graphite composite for electrochemical energy storage. J. Power Sources 275, 688-693 (2015). doi:10.1016/j.jpowsour. 2014.11.061

38. H.R. Jung, W.J. Lee, Ag/poly(3,4-ethylenedioxythiophene) nanocomposites as anode materials for lithium ion battery. Solid State Ionics 187(1), 50-57 (2011). doi:10.1016/j.ssi.2010.12.019 
39. C.Z. Yuan, B. Gao, X.G. Zhang, Electrochemical capacitance of $\mathrm{NiO} / \mathrm{Ru}_{0.35} \mathrm{~V}_{0.65} \mathrm{O}_{2}$ asymmetric electrochemical capacitor. J. Power Sources 173(1), 606-612 (2007). doi:10.1016/j.jpow sour.2007.04.034

40. X. Guo, L. Li, X.H. Zhang, J.H. Chen, Platinum nanoparticles encapsulated in nitrogen-doped mesoporous carbons as methanoltolerant oxygen reduction electrocatalysts. Chemelectrochem 2, 404-411 (2015). doi:10.1002/celc.201402342

41. B. Guo, X. Wang, P.F. Fulvio, M. Chi, S.M. Mahurin, X.-G. Sun, S. Dai, Soft-templated mesoporous carbon-carbon nanotube composites for high performance lithium-ion batteries. Adv. Mater. 23(40), 4661-4666 (2011). doi:10.1002/adma.201102032
42. T. Yang, Z. Chen, H. Zhang, M. Zhang, T. Wang, Multifunctional $\mathrm{Cr}_{2} \mathrm{O}_{3}$ quantum nanodots to improve the lithium-ion storage performance of free-standing carbon nanofiber networks. Electrochim. Acta 217, 55-61 (2016). doi:10.1016/j.electacta.2016. 09.062

43. M. Zhang, Y. Li, E. Uchaker, S. Candelaria, L. Shen, T. Wang, G. $\mathrm{Cao}$, Homogenous incorporation of $\mathrm{SnO}_{2}$ nanoparticles in carbon cryogels via the thermal decomposition of stannous sulfate and their enhanced lithium-ion intercalation properties. Nano Energy 2(5), 769-778 (2013). doi:10.1016/j.nanoen.2013.01.009 